STUDI

FRANCESI

\section{Studi Francesi}

Rivista quadrimestrale fondata da Franco Simone

165 (LV | III) | 2011

LA RAPPRESENTAZIONE DELLA MADRE NELLA

LETTERATURA FRANCESE DEL NOVECENTO a cura di Dario Cecchetti e Michele Mastroianni

\title{
Andy Stafford, Photo-texts. Contemporary French Writing of the Photographic Image
}

\section{Francesca Lorandini}

\section{OpenEdition \\ Journals}

Edizione digitale

URL: http://journals.openedition.org/studifrancesi/5154

DOI: $10.4000 /$ studifrancesi.5154

ISSN: 2421-5856

Editore

Rosenberg \& Sellier

Edizione cartacea

Data di pubblicazione: 1 décembre 2011

Paginazione: 681

ISSN: 0039-2944

Notizia bibliografica digitale

Francesca Lorandini, «Andy Stafford, Photo-texts. Contemporary French Writing of the Photographic Image», Studi Francesi [Online], 165 (LV | III) | 2011, online dal 30 novembre 2015, consultato il 10 janvier 2021. URL: http://journals.openedition.org/studifrancesi/5154 ; DOI: https://doi.org/10.4000/ studifrancesi.5154

Questo documento è stato generato automaticamente il 10 janvier 2021.

\section{(†) $\ominus$

Studi Francesi è distribuita con Licenza Creative Commons Attribuzione - Non commerciale - Non opere derivate 4.0 Internazionale. 


\title{
Andy Stafford, Photo-texts. Contemporary French Writing of the Photographic Image
}

\author{
Francesca Lorandini
}

\section{NOTIZIA}

ANDY STAFFORD, Photo-texts. Contemporary French Writing of the Photographic Image, Liverpool, Liverpool University Press, 2010 («Contemporary French and Francophone Cultures», 14), pp. 246.

1 A. Stafford analizza quella singolare forma intermediale che è il photo-text, postulando l'esistenza di un nuovo campo di studio che si apre nel dialogo tra fotografia e scrittura. Il libro si concentra sugli anni del New World Order postcomunista: è in questo periodo infatti che acquisisce un'importanza notevole, in fotografia, il passaggio da un atteggiamento meramente documentario a una sorta di scetticismo rappresentativo (si pensi alla distanza tra Cartier-Bresson e l'«exercice politique d'une pratique artistique» - p. 12 - sviluppato da Thomas Hirschhorn).

La tesi di Stafford è chiara: nel photo-text testo e immagine coesistono in un'interrogazione reciproca e concorrono in egual misura alla resa estetica, generando una dimensione riflessiva e resistenziale di natura politica. Al di là della questione autoriale infatti (il rapporto tra scrittore e fotografo), il filo conduttore che percorre l'intera rassegna di photo-texts è il legame da questi intrattenuto con la Storia: problematizzando la dicotomia tra verità e finzione, ognuno di essi rivisita la storia istituzionalizzata attraverso uno sguardo obliquo sul passato. Ciò è evidente nel cortocircuito tra scatto e commento nei lavori di Philippe Tagli sulla banlieue parigina; nell'invito alla rivoluzione permanente che Bernard Noël legge nelle immagini d'archivio della Commune; nelle tracce della memoria cancellata del bagne che Chamoiseau e Hammadi ritrovano nella Guyana francese o nella dialettica tra fotografia 
e scrittura che Leïla Sebbar riconosce parallela a quella tra gaze e voile nelle celebri immagini di donne algerine di Marc Garanger. Ma altrettanto vale per la dimensione vernacolare che Tahar Ben Jelloun ritrova nella fotografie della brulicante Fez diurna e nell'opacità notturna della medina; per la riflessione che Anne-Marie Garat sviluppa in Photos de famille sull'iconografia della cultura occidentale; per le strategie retoriche impiegate da Régis Debray nell'interrogazione del medium fotografico stesso o per la fenomenologia dell'erranza realizzata da Raymond Depardon in un gioco polisemico di rimandi tra testo e immagine.

3 Nonostante qualche eccesso di erudizione, il libro, corredato da otto illustrazioni in bianco e nero, esamina con cura una forma artistica che ha visto un importante sviluppo in Francia negli ultimi decenni (basti pensare all'attenzione crescente manifestata dalle maggiori case editrici o all'apertura di una collana come «Traits et portraits» da parte di Mercure de France), attraverso un discorso teorico in dialogo con i classici (R. Barthes, S. Sontag, G. Freund) e con i contemporanei (W.J.T Mitchell, A. Rouillé, F. Soulages, J. Rancière, H. Damisch). Stafford, inoltre, concentrandosi su lavori di non-fiction, riesce bene a mettere in evidenza la sfida che questa particolare forma saggistica si propone agli albori del nuovo millennio: evitare tanto le pretese testimoniali del reportage quanto la funzione decorativa di quello che $\mathrm{i}$ francesi chiamano beau livre e gli inglesi - con più mordente - coffee table book. 